Surgery and Epilepsy in Progress in Pediatric Neurology, JG Millichap ed, 1991, pp 131-138).

\title{
POST-OPERATIVE EPILEPSY RECURRENCE
}

Post-operative seizures occurred in 72 patients after resective procedures for epilepsy at The Epilepsy Surgery Program, Departments of Neurology and Neurological Surgery, The Cleveland Clinic Foundation, Cleveland, $\mathrm{OH}$. The patients ranged in age from 2-47 years (mean 26 years). Temporal lobe resection had been performed in $74 \%$ and extratemporal resections in $26 \%$. The post-operative seizures were similar to preoperative patterns in $74 \% .86 \%$ of patients had their first seizure occurrence within 1 year of surgery. Of 21 patients with recurrences in the first week after operation, none had subtherapeutic antiepileptic drug levels. Those with first recurrences from 1 week to more than 1 year after operation had subtherapeutic levels in 10 to $50 \%$ of patients. One-half of the patients had more than 1 seizure a month. In temporal resection patients half of the recurrences were controllable, whereas patients with extratemporal resections had a greater likelihood $(96 \%)$ of persistent seizures and seizures became intractable in $74 \%$. The age at operation, the duration of epilepsy, and associated structural lesions did not influence the likelihood of persistent seizures or intractability after recurrence. (Wingkun EC, Awad IA et al. Natural history of recurrent seizures after resective surgery for epilepsy. Epilepsia Nov/Dec 1991; 32:851-856.) (Reprints: Dr. Awad, Department of Neurological Surgery, Cleveland Clinic Foundation, 9500 Euclid Ave., Cleveland, OH 44195.)

COMMENT. After the first seizure recurrence there appears to be a predictable trend of persistent seizures over the next 5 years. A decrease in seizure recurrence in the first 3 years after the first episode of relapse may reflect a more careful attention to antiepileptic drug therapy. Seizures recurring within 1 week postoperatively are not benign and have the same likelihood of subsequent intractability as other recurrent seizures in the first postoperative year. The outcome of resective surgery in extratemporal regions was less encouraging than temporal resections. If seizure recurrences are delayed until after the first postoperative year the outlook is better and seizures rarely become intractable. Changes in antiepileptic drugs, dosages or poor compliance may be important factors in recurrence of seizures. The maturing epileptogenicity at the scar of a previous resection does not appear to contribute to intractability of seizures but may be a factor in later recurrences.

Details of the anticonvulsant drug regimens used before and after operation were not provided and the comparative efficacy of phenytoin and carbamazepine in postoperative epilepsy would be of interest. A comparative study of the cognitive effects of phenytoin and carbamazepine in healthy young adults has shown no significant clinical differences between the 2 drugs. (Meador KJ et al. Comparative cognitive effects of carbamazepine and phenytoin in healthy adults. Neurology, 1991; $41: 1537$. 